Bernhard Lübbers

\title{
Die Staatliche Bibliothek Regensburg: eine Regionalbibliothek im Strukturwandel
}

Zusammenfassung: Der Beitrag hebt darauf ab, dass für Regionalbibliotheken neben der digitalen Bereitstellung der Bestände auch die wissenschaftliche Komponente nicht vernachlässigt werden darf.

Schlüsselwörter: Strukturwandel, Digitalisierung, Google books, Wissenschaftlichkeit, Wissenschaftlicher Bibliothekar

\section{The Regional State Library Regensburg: A regional library facing far-reaching change}

Abstract: The essay shows that especially for regional libraries it is not only important to present digitized material, but to support and also be actively involved in research.

Keywords: re-engineering, digitization, google books, research, research librarian

Dr. Bernhard Lübbers: b.luebbers@sb-regensburg.de

\section{Einleitung}

Bibliotheken weltweit und demzufolge natürlich auch Regionalbibliotheken wie die Staatliche Bibliothek Regensburg sind mit einem „globalen Megatrend“ konfrontiert: der Digitalisierung. Die Welt erlebt bekanntlich seit einigen Jahren einen grundstürzenden Medienbruch, der in seinem Ausmaß wohl nur mit anderen großen Medienbrüchen der Vergangenheit zu vergleichen ist, etwa mit dem Übergang von der Handschrift zum gedruckten Buch in der zweiten Hälfte des 15. Jahrhunderts. ${ }^{1}$ Doch während damals im Wesentlichen lediglich die Welt

1 Einer der ersten, der auf diese „ganz erstaunliche[n] strukturelle[n] Parallelen“ aufmerksam gemacht hat, war Giesecke, Michael: Der Buchdruck in der frühen Neuzeit. Eine historische Fallstudie über die Durchsetzung neuer Informations- und Kommunikationstechnologien. Frank- 
der Gelehrten betroffen war, verändert die digitale Revolution heute nahezu alle Lebensbereiche und hat nicht nur in Bibliotheken zu erheblichen Verschiebungen geführt. Sowohl die Publikations- und Distributionswege für wissenschaftliche Informationen als auch das Nutzungsverhalten haben sich nachhaltig verändert und verändern sich weiterhin dramatisch. ${ }^{2}$

\section{Geschichte und Bestandsprofil}

Die Staatliche Bibliothek Regensburg wurde im Jahr 1816 aus den Resten der 1810 nach dem Übergang der ehemaligen Reichsstadt Regensburg an das Königreich Bayern aufgelösten Bibliotheken sämtlicher geistlichen und weltlichen Institutionen der Stadt gebildet. ${ }^{3}$ Zunächst im alten Bibliotheksgebäude der Reichsstadt Regensburg im sogenannten Neuen Waaggebäude am Haidplatz untergebracht, wo sich seit 1783 die reichsstädtische Stadtbibliothek befunden hatte, wurden 1875/76 Räumlichkeiten im ehemaligen reichsstädtischen Gymnasium poeticum an der Regensburger Gesandtenstraße, im Herzen der Altstadt, bezogen, wo sich die Bibliothek bis heute befindet. Die Staatliche Bibliothek Regensburg fungiert heute als traditionsreiche wissenschaftliche Regionalbibliothek für den bayerischen Regierungsbezirk Oberpfalz und partizipiert als Zweitexemplarbibliothek am bayerischen Pflichtstückegesetz von $1986^{4}$. Sie zählt überdies zum Kreis von insgesamt zehn regionalen Staatlichen Bibliotheken im Freistaat Bayern und

furt a. M. 1991, S. 24. Inzwischen hat das Buch mehrere Neuauflagen erfahren und ist auch als Taschenbuch, bislang allerdings noch nicht als E-Book erhältlich.

2 Teile dieses Beitrages finden sich bereits bei: Lübbers, Bernhard: Tradition und Dynamik. Die Staatliche Bibliothek Regensburg. Eine Regionalbibliothek als Akteur der regionalen Geschichtskultur. In: Memminger, Josef (Hg.): Überall Geschichte! Der Lernort Welterbe - Facetten der Regensburger Geschichtskultur. Regensburg 2014, S. 93-115.

3 Vgl. zur Gründung der Bibliothek Hauschka, Ernst R.: Zur Gründung der Staatlichen Bibliothek Regensburg in den Jahren 1816/17. In: Ostbairische Grenzmarken 7 (1964/65), S.125-133. Zu den aufgelösten Bibliotheken vgl. Drucker, Michael: Regensburger Bibliotheken: Schicksale zwischen Reichsstadtzeit und Königreich. In: Schmid, Peter; Unger, Klemens (Hg.): 1803. Wende in Europas Mitte. Vom feudalen zum bürgerlichen Zeitalter. Regensburg 2003, S.187-208, sowie Knedlik, Manfred; Lübbers, Bernhard (Hg.): Die Regensburger Bibliothekslandschaft am Ende des Alten Reiches (Kataloge und Schriften der Staatlichen Bibliothek Regensburg 5). Regensburg 2011.

4 Gesetz über die Ablieferung von Pflichtstücken. Pflichtstückegesetz (PflStG) vom 6. August 1986. Veröffentlicht: Bayerisches Gesetz- und Verordnungsblatt 1986, S. 216f., online unter: http://www.bsb-muenchen.de/fileadmin/imageswww/pdf-dateien/abteilungen/Pflichtstueckegesetz.pdf [Zugriff, auch bei allen folgenden Links: 07.04.2014]. 
ist der Bayerischen Staatsbibliothek in München nachgeordnet. ${ }^{5}$ Im Jahr 2008 wurde zudem eine Kooperationsvereinbarung zwischen der Staatlichen Bibliothek und der Universitätsbibliothek Regensburg abgeschlossen, die das Profil der Staatlichen Bibliothek Regensburg als Regionalbibliothek für Regensburg und die Oberpfalz nachhaltig schärft. ${ }^{6}$ Sie nennt in ihrem Gesamtbestand von etwa 360.000 Medien mehr als 90.000 bibliographische Einheiten mit Erscheinungsjahr vor 1800 ihr Eigen, davon mehr als 1.100 Inkunabeln, sowie ca. 900 Handschriften, 750 Autographen und mehr als 7.000 Altkarten einer bedeutenden Privatsammlung, die 1787 an die damalige Stadtbibliothek der Reichsstadt Regensburg gelangte, darunter viele Unika. Sie ist damit eine der wichtigsten Institutionen für die historische Überlieferung der traditionsreichen Weltkulturerbestadt Regensburg, zumal sich auch der gedruckte Bestand aus ehemals in Regensburg beheimateten Institutionen zusammensetzt. ${ }^{7}$ Viele herausragende und für die kulturelle Identität Regensburgs wichtige Stücke sind darunter. Ein wahrer Fundus ist dabei der Handschriftenbestand der Bibliothek. Dieser darf zu großen Teilen als Streubestand, gewissermaßen als das „Strandgut der Säkularisation“ gelten, das aus den verschiedensten Quellen den Weg in die Bibliothek fand.

\section{Digitalisierung}

Da sich das Benutzerverhalten insbesondere der nachwachsenden Generationen dramatisch verändert, ist es wichtig, gerade in diesem Bereich tätig zu werden

5 Seit Dezember 2012 gehört die bis 2011 fast ausschließlich städtisch finanzierte Staats- und Stadtbibliothek Augsburg als zehnte Bibliothek zu diesem Kreis. Vgl. hierzu den Webauftritt der regionalen Staatlichen Bibliotheken in Bayern: http://www.regionalbibliotheken-bayern.de sowie die Homepage der Bibliothek selbst: http://www.susb-augsburg.de/.

6 Vgl. hierzu Griebel, Rolf: Der Beginn einer strategischen Partnerschaft. Die Universitätsbibliothek und die Staatliche Bibliothek Regensburg schließen eine Kooperationsvereinbarung. In: Bibliotheksforum Bayern 3 (2009), S. 111-112, sowie Lübbers, Bernhard: Die Staatliche Bibliothek Regensburg schärft ihr Profil als wissenschaftliche Regionalbibliothek für Regensburg und die Oberpfalz. In: Die Oberpfalz. Heimatzeitschrift für den ehemaligen Bayerischen Nordgau 98 (2010), S. 65-67.

7 Details zum Bestand finden sich in: Die Staatliche Bibliothek in Regensburg. Benützungshinweise. Führer durch die Sammlung. Gesamtbibliographie. Regensburg [1967] sowie bei Steinmaus-Pollak, Angelika; Drucker, Michael: Art. Regensburg 1: Staatliche Bibliothek. In: Handbuch der historischen Buchbestände in Deutschland, Bd.12, hg. von Eberhard Dünninger. Hildesheim/Zürich/New York 1996, S. 222-229. Eine stets aktualisierte Übersicht wird auch auf der Website der Bibliothek geboten: http://www.staatliche-bibliothek-regensburg.de/index. php?id=42. 
und die Entwicklung der Staatlichen Bibliothek Regensburg hin zu einer Hybridbibliothek zu forcieren. ${ }^{8}$ Es werden dabei mehrere Strategien zugleich verfolgt: So erfolgt die Digitalisierung ausgewählter Bestände über den Service „EOD“ (Ebooks on demand) in Zusammenarbeit mit der Universitätsbibliothek Regensburg. Da der Bestand beider Institutionen im gemeinsam geführten Regensburger Katalog nachgewiesen ist, kann der Nutzer einfach und bequem einen Digitalisierungswunsch absetzen, sofern der gewünschte Titel aus urheberrechtlichen Erwägungen heraus überhaupt reproduziert werden darf.

Einen gänzlich anderen Ansatz verfolgt die Digitalisierung und Bereitstellung im „Schwerpunkt Regensburg“ der Bayerischen Landesbibliothek Online. ${ }^{9}$ Mehrere Kooperationspartner, das Stadtarchiv Regensburg, die Universitätsbibliothek Regensburg, die Staatliche Bibliothek Regensburg und die Bayerische Staatsbibliothek in München, haben sich hier zusammengeschlossen, um die disparate Überlieferungssituation Regensburgs sukzessive virtuell zusammenzuführen. Die Überlieferung kaum einer anderen Stadt stellt sich heute so fragmentiert dar. Daher ist es für die historisch arbeitenden wissenschaftlichen Disziplinen bis heute mit großem Aufwand verbunden, umfassende Forschungen zu Regensburg und den zahlreichen bedeutenden Institutionen, weltlichen wie geistlichen, zu betreiben. Weiterhin sind in einer zunehmend globalisierten Welt regional bedeutende Kulturgüter auch hochemotional „aufgeladen“. Das Wort „Beutegut“ wird in solchen Zusammenhängen oft sehr schnell aufgenommen. Auch Handschriften und seltene Drucke zählen zu diesen Zeugnissen regionaler Identität. Dafür muss man nicht erst an Island erinnern, wohin 1971 mehrere Handschriften aus Dänemark zurückkehrten, was zur ersten Live-Übertragung in der Geschichte des isländischen Fernsehens überhaupt führte und tausende Menschen auf die Straßen trieb. Diese genannten Gründe bewogen die Projektpartner, den „Schwerpunkt Regensburg“ innerhalb der Bayerischen Landesbibliothek Online ins Leben zu rufen. Es handelte sich um den ersten Regionalschwerpunkt in diesem Portal, weshalb dem Unternehmen zudem ein prototypischer Charakter zukam. Projektbasiert werden hier Teile der zerstreuten Regensburger Überlieferung virtuell wieder zusammengeführt und können so bequem von der Wissenschaft benutzt

8 Vgl. hierzu Lübbers, Bernhard: Kooperativ durchs digitale Zeitalter. Die Digitalisierungsstrategie der Staatlichen Bibliothek Regensburg. In: Siebert, Irmgard (Hg.): Digitalisierung in Regionalbibliotheken (Zeitschrift für Bibliothekswesen und Bibliographie. Sonderband 107). Frankfurt am Main 2012, S. 291-302.

9 Vgl. Ball, Rafael; Lübbers, Bernhard: Regensburgs kulturelles Erbe erhält ein eigenes Portal. In: Bibliotheksforum Bayern 4 (2010), S. 259-260, online unter: http://www.bsb-muenchen.de/ fileadmin/imageswww/pdf-dateien/bibliotheksforum/2010-4/BFB_0410_10_Ball-Luebbers_ V02.pdf. 
werden. Ein markantes Beispiel für die Vorteile dieser Vorgehensweise bietet die virtuelle Rekonstruktion der Bibliothek der Reichsstadt Regensburg, welche unmittelbar nach 1810, dem Jahr des Übergangs Regensburgs an das Königsreich Bayern, aufgelöst worden war. ${ }^{10}$ Der Kern dieser Sammlung, die Ratsbibliothek ${ }^{11}$, darf als der am frühesten bezeugte Vertreter dieses Bibliothekstyps angesehen werden und zählte über Jahrhunderte zu den Attraktionen der reisenden Bildungsschicht ${ }^{12}$. Noch Kronprinz Ludwig, der spätere bayerische König (1825-1848), besuchte im Januar 1810 die seit 1783 vereinigte Stadtbibliothek und bewunderte ihre Schätze. ${ }^{13}$ Handschriften und Drucke dieser Bibliothek sind heute verstreut, die allermeisten gelangten in die Bayerische Staatsbibliothek nach München, einige blieben jedoch in Regensburg, darunter etwa nahezu sämtliche Altkataloge. In der Bayerischen Landesbibliothek Online sollen nun die bekannten Handschriften virtuell zusammengeführt werden, um der Wissenschaft den Zugriff auf diesen reichen Fundus zu erleichtern. In einem ersten Schritt wurden im Oktober 201039 mittelalterliche und frühneuzeitliche Handschriften aus der ehemaligen reichsstädtischen Bibliothek Regensburg, die sich heute im Bestand der Bayerischen Staatsbibliothek in München befinden, digital zugänglich gemacht. Es handelt sich hierbei um die ältesten und wertvollsten Handschriften der ehemaligen Stadtbibliothek Regensburg. Seitdem wird das Angebot sukzessive erweitert und ergänzt. Im Zuge der Bemühungen um den „Schwerpunkt Regensburg“ in der Bayerischen Landesbibliothek Online, hat sich auch die Unternehmensleitung des Mittelbayerischen Verlages KG entschlossen, zusammen mit dem Stadtarchiv

10 Vgl. hierzu Lübbers, Bernhard: Johann Baptist Bernhart (1759-1821) und seine Regensburger Bibliotheksreise im Winter 1811/12. In: Appl, Tobias; Köglmeier, Georg (Hg.): Regensburg, Bayern und das Reich. Festschrift für Peter Schmid zum 65. Geburtstag. Regensburg 2010, S. 597-617.

11 Lübbers, Bernhard: Regensburg hat die älteste Ratsbibliothek Deutschlands. Eine kleine Sensation für die bayerische Historiographie. In: Regensburger Almanach 2007, S. 86-91, sowie Ders.: Eine unbeachtete deutsche Chronik des 14. Jahrhunderts aus der Reichsstadt Regensburg. Eines der ältesten Zeugnisse geschichtlicher Prosa-Darstellung in deutscher Sprache aus Bayern. In: Zeitschrift für bayerische Landesgeschichte 71 (2008), S. 707-745.

12 Vgl. etwa Dünninger, Eberhard: Regensburger Bibliotheken im Spiegel von Reiseberichten des 18. Jahrhunderts. In: Regensburger Almanach 1988, Regensburg 1987, S.145-151 (wieder abgedruckt in: Ders.: Oberpfalz und Regensburg. Kultur- und Lebensbilder. Regensburg 1998, S. 72-79).

13 Zur vereinigten Stadtbibliothek vgl. Drucker, Michael: Bürger und Bücher. Die Bibliothek der Reichsstadt Regensburg. Ausstellung im ehemaligen reichsstädtischen Bibliothekssaal, heute Sitzungssaal des Verwaltungsgerichts Regensburg 9.6.-25.6.1999 und in der Staatlichen Bibliothek Regensburg 1.7.-17.7.1999. Regensburg 1999, S. 5f. Zum Besuch des Kronprinzen: Lübbers, Bernhard: König Ludwig I. und Regensburg. Freund und Förderer. In: Regensburg bayerisch 1810. Regensburger Almanach 2010, S. 20-29. 
Regensburg, der Staatlichen Bibliothek Regensburg sowie der Bayerischen Staatsbibliothek München, die Stadtausgabe Regensburg der Mittelbayerischen Zeitung der Jahre 1945 bis 2005 digital über den Schwerpunkt Regensburg bereitzustellen. Erste Ergebnisse wurden zu Beginn des Jahres 2012 bereits der Öffentlichkeit präsentiert. ${ }^{14}$ Viele Teilprojekte könnten hier noch erwähnt werden, etwa die Aufführungsbücher der Regensburger Domspatzen ${ }^{15}$ oder auch die Totenrotelsammlung des ehemaligen Benediktinerstiftes St. Emmeram ${ }^{16}$, aus Platzgründen sollen aber diesen wenigen Hinweisen genügen.

\section{Der Google-Deal der Bayerischen Staatsbibliothek und die Einbeziehung der Staatlichen Bibliothek Regensburg}

Wichtigstes Element in der Digitalisierungsstrategie der Staatlichen Bibliothek Regensburg ist die Massendigitalisierung. Die Bayerische Staatsbibliothek digitalisiert bereits seit 2007 große Teile ihres urheberrechtsfreien Bestandes mit Google. ${ }^{17}$ In diese Zusammenarbeit wurde auch die Staatliche Bibliothek Regensburg mit ausgewählten Beständen einbezogen. Oberste Kriterien bei der Auswahl der Bestände waren, dass die Bücher nicht nur urheberrechtsfrei, sondern auch in der Bayerischen Staatsbibliothek nicht oder zumindest nicht in einer identischen Ausgabe vorhanden sind. Dazu wurde ein Katalogabgleich erstellt, der ergab, dass die Staatliche Bibliothek Regensburg 70.000 Bände besitzt, die beide genannten Merkmale aufweisen. Im November 2012 wurde mit der Massendigitalisierung begonnen. Insgesamt sollen also 70.000 Bände aus dem Bestand der SBR mit rund 14 Millionen Seiten digital verfügbar werden. Anfang 2014 war eine wichtige Zwi-

$14 \mathrm{http} / /$ www.bayerische-landesbibliothek-online.de/mittelbayerische. Zahlreiche weitere Projekte wären hier noch zu nennen, da jedoch nur begrenzt Raum zur Verfügung steht, muss ein lediglich summarischer Verweis genügen. Eine Übersicht der in Arbeit befindlichen Projekte im Rahmen der Bayerischen Landesbibliothek Online findet sich auf dieser Website: http://www. bayerische-landesbibliothek-online.de/regensburg-projekte.

$15 \mathrm{http}: / /$ www.bayerische-landesbibliothek-online.de/domspatzen.

$16 \mathrm{http}: / /$ www.bayerische-landesbibliothek-online.de/totenroteln-emmeram.

17 Vgl. hierzu grundlegend: Ceynowa, Klaus: Der BSB-Google-Deal. Eine Million Bücher der Bayerischen Staatsbibliothek online. In: Bibliotheks-Magazin 1 (2008), S. 3-7; Ders.: Massendigitalisierung für die Wissenschaft. Zur Digitalisierungsstrategie der Bayerischen Staatsbibliothek. In: Griebel, Rolf; Ceynowa, Klaus (Hg.): Information - Innovation - Inspiration. 450 Jahre Bayerische Staatsbibliothek. München 2008, S. 241-252, hier besonders S. 245-248. 
schenmarke - 50.000 Bände online - erreicht. ${ }^{18}$ Eine - gerade für eine verhältnismäßig kleine Bibliothek - gewaltige Zahl. Große Teile des gedruckten Erbes der ehemaligen Reichsstadt Regensburg werden damit weltweit sichtbar gemacht. ${ }^{19}$

Ziel aller genannten Anstrengungen muss es sein, mittel- bis langfristig den gesamten physisch vorhandenen urheberrechtsfreien Bestand der Staatlichen Bibliothek Regensburg auch in digitaler Form anbieten zu können.

Bei allen Bemühungen um die digitale Bereitstellung der Bestände spielt neben der deutlich verbesserten Benutzbarkeit, auch der Aspekt der Bestandserhaltung eine tragende Rolle, der so auch in der Bayerischen Verfassung verankert ist. „Der Staat schützt die natürlichen Lebensgrundlagen und die kulturelle Überlieferung“, steht dort zu lesen (Art. 3, Abs.1, Satz 2). Digitalisierte Bestände werden erfahrungsgemäß nicht mehr so oft physisch benutzt; sie werden somit geschützt und die Überlieferungschance - auch in ihrer Materialität - für kommende Generationen deutlich verbessert. ${ }^{20}$

\section{Sichtbarkeit}

Seit die Massendigitalisierung mit Google begonnen hat, haben sich auch die Anfragen auf die sichtbaren digitalen Bestände der Staatlichen Bibliothek Regensburg signifikant erhöht. Dass der oft bemühte Topos, dass digitale Bestände weltweit Sichtbarkeit erzeugen, nicht nur eine schön klingende Aussage ist, zeigt die Herkunft der Anfragen. Waren vor Beginn der Massendigitalisierung Anfragen zu Beständen der Staatlichen Bibliothek Regensburg aus Übersee eher die Ausnahme, sind seither Auskunftswünsche aus Tokio, Brisbane oder San Francisco keine Seltenheit mehr. Ein schlagender Beweis dafür, dass die Digitalisierung urheberrechtsfreier Bestände nicht bloß für einige wenige historisch Interessierte einen Mehrwert erbringt.

18 Vgl. die Pressedokumentation zu diesem Zwischenschritt auf: http://www.staatliche-biblio thek-regensburg.de/index.php?id=47.

19 Vgl. Wittl, Wolfgang: 14 Millionen Seiten geballtes Wissen. Die Schätze der Regensburger Staatsbibliothek werden gescannt und stehen dann der Welt zur Verfügung. In: Süddeutsche Zeitung vom 24. April 2013, S. 38, sowie Ceynowa, Klaus; Lübbers, Bernhard: Ritterschlag für den Wissenschaftsstandort Bayern. Der Google-Deal der Bayerischen Staatsbibliothek und die Einbeziehung der Staatlichen Bibliothek Regensburg. In: Bibliotheksforum Bayern 7 (2013), S. 166-167. 20 Vgl. hierzu die Beobachtungen, die an der Bayerischen Staatsbibliothek München getätigt wurden: Baumgartner, Martin; Hilpert, Wilhelm: Halbzeit - ohne Pause. Stand und Erkenntnisse der industriellen Massendigitalisierung an der Bayerischen Staatsbibliothek. In: b.i.t.online 14 (2011), S. 133-138, hier insbesondere S. 136. 


\section{Wissenschaftlichkeit}

Für eine Regionalbibliothek ist es sicherlich nicht nur wichtig, sondern unerlässlich, Bestände digital zur Verfügung zu stellen. Für die Zukunftsfähigkeit einer Bibliothek dieses Zuschnitts alleine reicht das aber nicht aus. Unter den vielfältigen Aufgaben, welche wissenschaftliche Bibliothekare heute wahrnehmen, ${ }^{21}$ ist die Annäherung an die Wissenschaft ein weiterer möglicher Weg, diese Häuser zukunftsfähig zu machen. ${ }^{22}$ Gewährsmann ist dafür sicherlich der große Paul Raabe $^{23}$, der sich in einem Interview einmal zu diesem Themenfeld geäußert hat: „Ich kann mir den wissenschaftlichen Bibliothekar in leitenden Funktionen nicht vorstellen ohne eine wissenschaftliche Betätigung. Denn es ist ja die wissenschaftliche Neugier, die auch nötig ist, wenn man Wissenschaftler, die eine Bibliothek benutzen wollen, anleiten und zu den Büchern hinführen soll. [...] denn der Bibliothekar soll ja schließlich ein Partner des Wissenschaftlers sein, und dieses kann er nur sein, wenn er auch selbst wissenschaftlich tätig ist.“24 Ähnlich äußert sich mit S. Ranganathan ${ }^{25}$, auch einer der weltweit bekanntesten Bibliothekare.

Dass dies besonders im regionalen Kontext wichtig, ja unabdingbar ist, steht nach unserer Auffassung außer Frage. Gerade hier ist der Schulterschluss mit der Wissenschaft essentiell. Zudem kann die regionale Identitätsbildung, die der wissenschaftlichen Untermauerung bedarf, als Gegenentwurf, ja nachgerade als Antwort auf die zunehmenden, alle Lebensbereiche erfassenden Auswirkungen der Globalisierung gelten, darüber sind sich weite Teile geisteswissenschaftlicher Forschung einig. ${ }^{26}$ Auch die Politik hat längst das Identifikations- und Integrationspotential regionaler Identitätskonzepte erkannt. Nicht ohne Grund wurde

21 Ein guter, aktueller Überblick bei: http://www.igwbs.ch/berufsbild/.

22 Zur Berufsbilddebatte vgl. jetzt Siebert, Irmgard; Lemanski, Thorsten (Hg.): Bibliothekare zwischen Verwaltung und Wissenschaft. 200 Jahre Berufsbilddebatte (ZfBB. Sonderband 111). Frankfurt a. M. 2014.

$23 \mathrm{Zu}$ ihm vgl. den posthum erschienen Sammelband: Raabe, Paul: Tradition und Innovation. Studien und Anmerkungen zur Bibliotheksgeschichte (ZfBB. Sonderband 110). Frankfurt a. M. 2013, hier insbesondere den Nachruf von Georg Ruppelt, S. 287-291; Ders.: In Leibniz' und Lessings Fußstapfen, in: b.i.t.online 6 (2013), S. 310-313; ferner Bürger, Thomas: Das Ende von Bibliosibirsk. In Memoriam Paul Raabe. In: Zeitschrift für Bibliothekswesen und Bibliografie 60 (2013), S. 220-223.

24 Der Bibliothekar - Partner der Wissenschaft. Interview mit Paul Raabe. In: Die Auskunft 18 (1998), S. 325-333.

25 Ranganathan, S[hiyali] R[amamrita]: The five laws of Library Science. London 1931, S. 44.

26 Vgl. etwa Schmid, Alois: Hundert Jahre „Die Oberpfalz“. In: Die Oberpfalz 95 (2007) S. 4-11, hier: S. 11. Grundsätzlich: Böhret, Carl: Zukunftsmodell „Glokalisierung“. Speyer 2010. 
in Bayern nach der Landtagswahl 2013 ein Heimatministerium eingerichtet. ${ }^{27}$ Für den hier im Mittelpunkt stehenden Bereich der Bibliothekslandschaft wird das Heil ebenfalls in einer Spezialisierung gesehen. ${ }^{28}$ Doch genau darin sind Regional- und Landesbibliotheken bereits jetzt erfahren. Und damit nicht genug: Es handelt sich sogar um eine ihrer Stärken. Das in den letzten Jahren entwickelte Konzept des „embedded librarian“ erhält so möglicherweise eine andere, um eine regionale Komponente erweiterte Ausrichtung. Mit Paul Raabe kann man also leicht abgewandelt sagen: „Ich kann mir den wissenschaftlichen Bibliothekar in leitenden Funktionen an einer Regional- und Landesbibliothek nicht vorstellen ohne eine wissenschaftliche Betätigung. “29 Dabei ist das kein nettes „Nice-tohave“, sondern gerade für kleinere Häuser überlebenswichtig. Dies kann ein Weg sein, auch Einrichtungen ohne große Ausstattung als Forschungsbibliotheken zu etablieren. ${ }^{30}$ Dem Autor ist dabei durchaus bewusst, dass sich nicht alle Kolleginnen und Kollegen gleichermaßen diesem Feld widmen können. ${ }^{31}$ Dennoch sollten wir versuchen, uns verstärkt auf die wissenschaftlichen Tugenden zu besinnen. Es handelt sich dabei um einen wichtigen, kaum zu unterschätzenden Beitrag, um gerade den Bibliothekstypus „Regionalbibliothek“ zukunftsfähig zu machen.

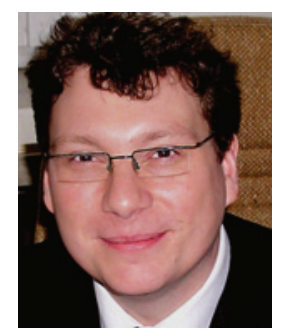

Dr. Bernhard Lübbers

Staatliche Bibliothek Regensburg

Gesandtenstraße 13

93047 Regensburg

E-Mail: b.luebbers@sb-regensburg.de

27 Vgl. bereits die Rede Horst Seehofers anlässlich des Tages der Heimat am 17. Mai 2009 in Neumarkt in der Oberpfalz, online unter: http://www.bayern.de/Anlage10248885/Bayerischer\%20 Heimattag\%202009.pdf.

28 Vgl. Kempf, Klaus: Der Sammlungsgedanke im digitalen Zeitalter. L’idea della collezione nell'età digitale (Lectio Magistralis in Biblioteconomia 6). Fiesole 2013, S. 53.

29 Siehe oben bei Anm. 23.

30 Vgl. hierzu Siebert, Irmgard: Die Zukunft liegt in der Vergangenheit. Historische Bibliotheken auf dem Weg zu Forschungsbibliotheken. In: Bibliothek, Forschung und Praxis 37 (2013) S. 78-90.

31 Klassisch sind die Ausführungen von Max Weber hierzu: Weber, Max: Wissenschaft als Beruf. In: Ders.: Schriften 1894-1922. Ausgewählt und hg. von Dirk Kaesler. Stuttgart 2002, S. 474-513. 